Photoacoustic studies on multilayer dielectric coatings

This article has been downloaded from IOPscience. Please scroll down to see the full text article.

1993 J. Phys. D: Appl. Phys. 26836

(http://iopscience.iop.org/0022-3727/26/5/017)

View the table of contents for this issue, or go to the journal homepage for more

Download details:

IP Address: 117.211.83.202

The article was downloaded on 29/10/2011 at 07:18

Please note that terms and conditions apply. 


\title{
Photoacoustic studies on multilayer dielectric coatings
}

\author{
Annieta Philip, P Radhakrishnan, V P N Nampoori and C P G \\ Vallabhan
}

Physics Department, Cochin University of Science and Technology, Cochin 682022, India

Received 8 April 1992, in final form 29 October 1992

\begin{abstract}
Non-destructive testing of multilayer dielectric coatings $\left(\mathrm{SiO}_{2} / \mathrm{TiO}_{2}\right.$ structure) has been carried out using the photoacoustic technique. This technique makes use of a $10 \mathrm{~mW}$ He-Ne laser, a photoacoustic cell and a lock-in amplifier. The chopped He-Ne laser beam is allowed to fall on the sample placed in a photoacoustic cell. The acoustic signals thus generated are detected using a microphone and the resulting output is processed by a lock-in amplifier. The amplitude and phase of the signals were measured as a function of the chopping frequency. Striking step-like variations are observed in the phase against frequency plot which clearly reveals the different layers present in the multilayer structure.
\end{abstract}

\section{Introduction}

The photoacoustic (PA) effect has been proven to be an effective and sensitive tool in studying optical and thermal properties of solids, liquids and gases. By using the PA technique one can obtain quantitative information on the sample properties such as the thermal diffusivity, optical absorption coefficient or thickness of a thin film specimen. In addition, by changing the chopping frequency of incident radiation, it is also possible to obtain a depth profile analysis of some of these properties. At high chopping frequencies, information about the sample near the surface is obtained while at low chopping frequencies, sample properties from deeper within become accessible. This is a feature unique to the PA technique. Another important aspect of the technique is its capability to measure the optical absorption of opaque samples.

The depth profiling capability of the PA technique can be exploited advantageously to study multilayer dielectric thin films coated on a substrate. Such periodic multilayers are extensively used as optical filters and laser mirrors. Ageing of multilayers will sometimes destroy the quality due to interlayer diffusion. The PA technique can thus effectively monitor the number of layers coated or it can be used to check whether there is a reduction in the effective number of layers due to such a diffusion process. The present paper deals with this aspect of the PA technique taking two representative multilayer thin film structures, namely 13 and 7 layers of $\mathrm{SiO}_{2}$ and $\mathrm{TiO}_{2}$ coated alternately on quartz substrates which act as highly reflecting mirrors at $632.8 \mathrm{~nm}$.

in order to understand the principle of depth profil- ing using the PA technique, consider a double layer thin film structure with a thin non-absorbing top layer and an optically and thermally thin bottom layer. The photoacoustic signal of such a structure is given by (Adam and Kirkbright 1977)

$$
q \sim \frac{\beta I_{0}}{c \omega \mu \rho} \exp (-x / \mu) \cos \left(\omega t-\frac{x}{\mu}-\frac{\pi}{4}\right)
$$

where $\beta$ is the absorption coefficient of the top layer, $\rho$ the density, $c$ the specific heat, $\omega$ the chopping frequency, $\mu$ the thermal diffusion length of the bottom layer and $x$ is the thickness of the upper layer. It is seen that the presence of a top layer causes a decrease in the signal and introduces a phase lag

$$
\psi=x / \mu \text {. }
$$

The additional phase lag $\psi$ in the PA signal will thus depend on the thickness of the top layer and the phase of the signal is quite sensitive to the presence of a boundary layer in a layered structure. This property of the PA technique was exploited by Adam and Kirkbright to determine the thickness of a polymer film on a copper substrate, while Baumann and Tilgner (1985) used the technique to determine the thickness of a buried layer. In addition, Mandelis et al (1979) discussed the utility of PA measurements in evaluating other material parameters. However, in all the reported studies the number of layers employed is very few (two or three). A detailed account of PA depth profiling has been given by Tam (1986). In the present paper we demonstrate the feasibility of the PA technique to characterize a stack of multilayer films. 


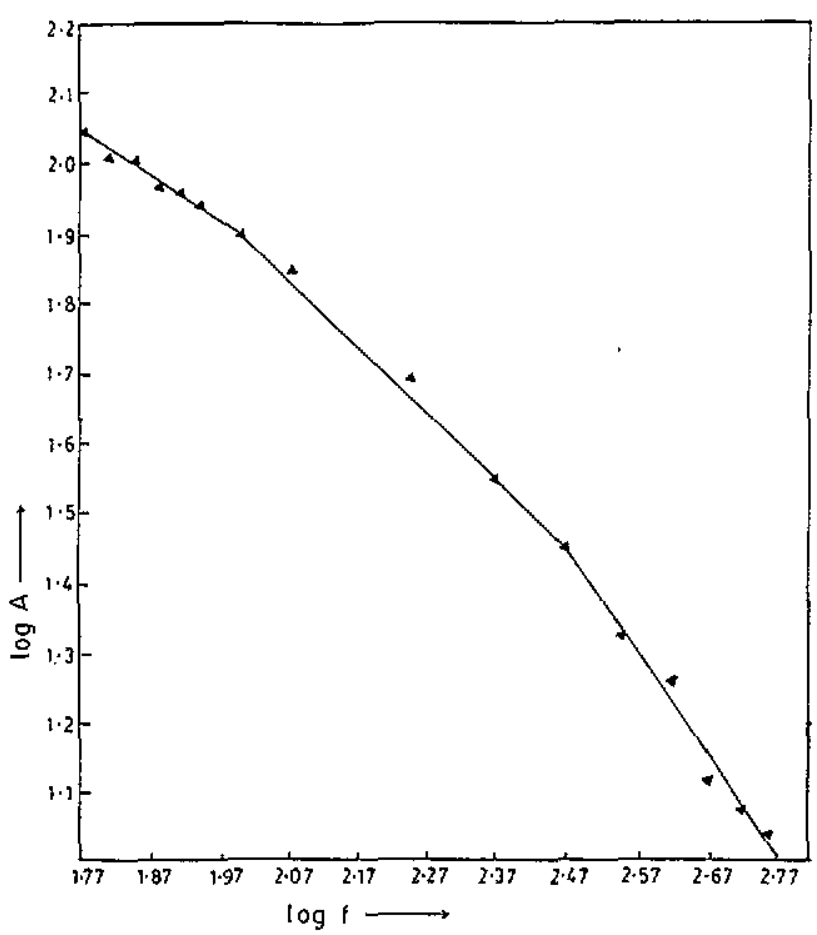

Figure 1. Log frequency against log amplitude for carbon black.

\section{Experimental set-up}

The photoacoustic cell used in the present study is a non-resonant type. The PA signal was detected using a sensitive microphone (Knowles-1834), the output of which was fed to a lock-in amplifier (EG \& G Model 5101 ) in which the phase of the signal can be measured with an accuracy of $\sim 1^{\circ}$. A $10 \mathrm{~mW}$ He-Ne laser (Spectra Physics) was used as the excitation source. The laser beam was modulated using a mechanical chopper and the phase and amplitude of the PA signal were measured as a function of the chopping frequency. The response of the PA cell to the incident radiation was evaluated using carbon black as the sample (figures 1 and 2). Samples having 13 and 7 layers $(\lambda / 4$ thick at $632.8 \mathrm{~nm}$ ), stacked alternately with $\mathrm{SiO}_{2}$ and $\mathrm{TiO}_{2}$ films on quartz substrates were used in the present investigation. The entire experimentation was repeated with an auto setting lock-in amplifier (EG \& G Model 5208 ) giving a phase accuracy of $0.01^{\circ}$ to confirm the validity of the results and to ensure no instrumental dependence.

\section{Results and conclusion}

Figures 3 and 4 show the dependence of the amplitude $q$ and phase $\psi$ of the PA signal with modulation frequency of the laser beam. Striking step-like variations in the phase plot due to phase lag at layer boundaries are clearly seen, unlike in the case of corresponding slope changes in the frequency dependence of signal strength. It is to be noted that figures 1 and 2 do

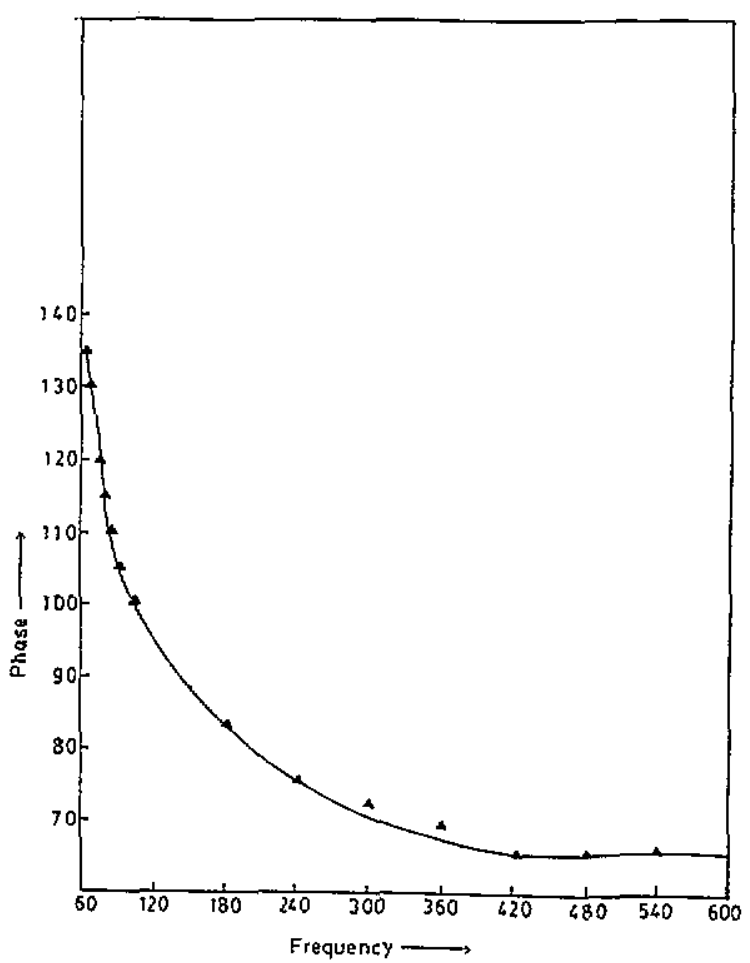

Figure 2. Frequency against PA signal phase for carbon black.

not reveal any type of discontinuities at the same resolution which rules out the role of any experimental artifact in the present results. Evidently the number of steps in the phase plot exactly matches the number of layers in the thin film structure. Furthermore, variations in the relative thickness of different layers are clearly discernible from the plot. This leaves no doubt as to the origin of these step-like variations in the phase-frequency plot of the PA signal. However, the relationship between the film thickness and phase lag need not be the simple form given in equation (2), apparently due to the complexity of PA generation in multilayer films of this type. Multiple reflections occurring in the thin film sublayer greatly enhance the effective optical absorption taking place in each layer and consequently one can no longer treat the sublayers as 'optically thin'. The effects of multiple reflections and interference on thermal waves (Opsal and Rosencwaig 1982) also have to be taken into account to obtain a correct theoretical picture of the actual phase lag produced from the multilayer structure. Detailed theoretical treatment of the PA effect in multilayer films is in progress. The scope of the present paper is essentially to enumerate the number of sublayers in a multilayer film which might indicate any possible interdiffusion, defect structures, etc, brought about during the deposition process and by ageing. The present work thus shows that the phase dependence of the chopping frequency of the PA signal is more sensitive than the dependence of PA signal amplitude on chopping frequency when a large numbet of layers are present in the thin film structure. Inadequacies in existing theories to deal with the PA 

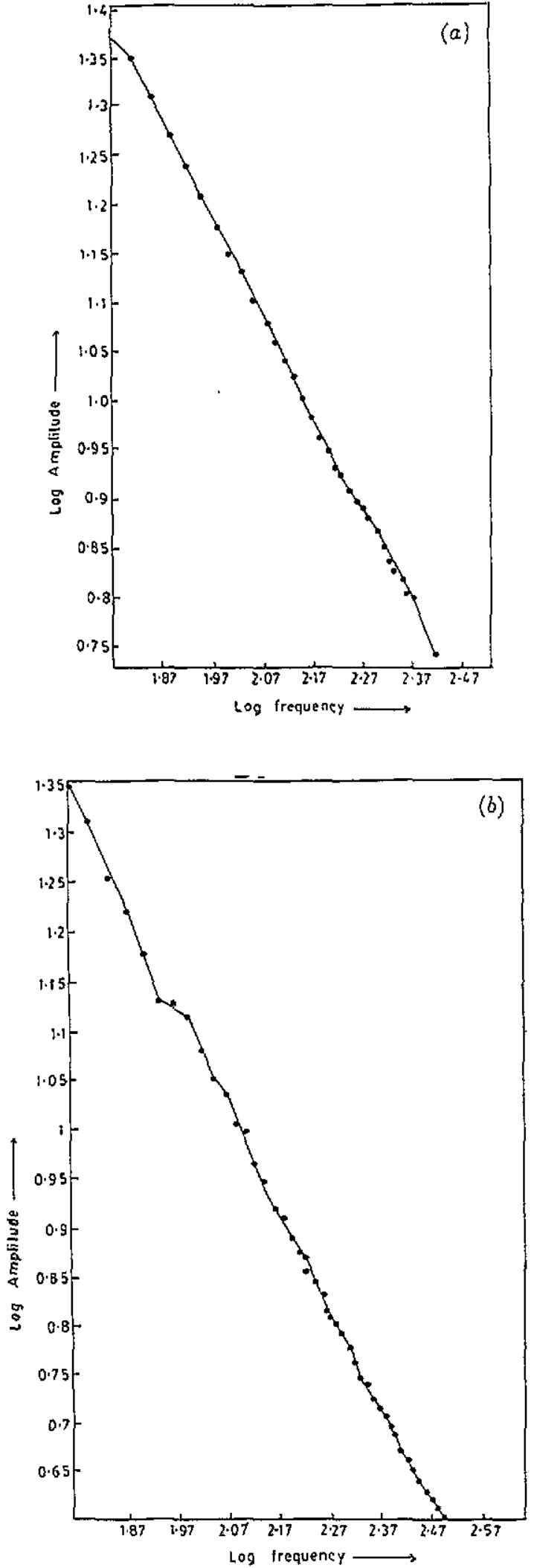

Figure 3. (a) Log frequency against log amplitude for 7 layer $\mathrm{SiO}_{2} / \mathrm{TiO}_{2}$ structure. (b) Log frequency against log amplitude for 13 layer $\mathrm{SiO}_{2} / \mathrm{TiO}_{2}$ structure.

effect in multilayer structures are also indicated.

In conclusion, it has been demonstrated that photoacoustics is an ideal technique to probe and analyse multilayer stacks non-destructively.
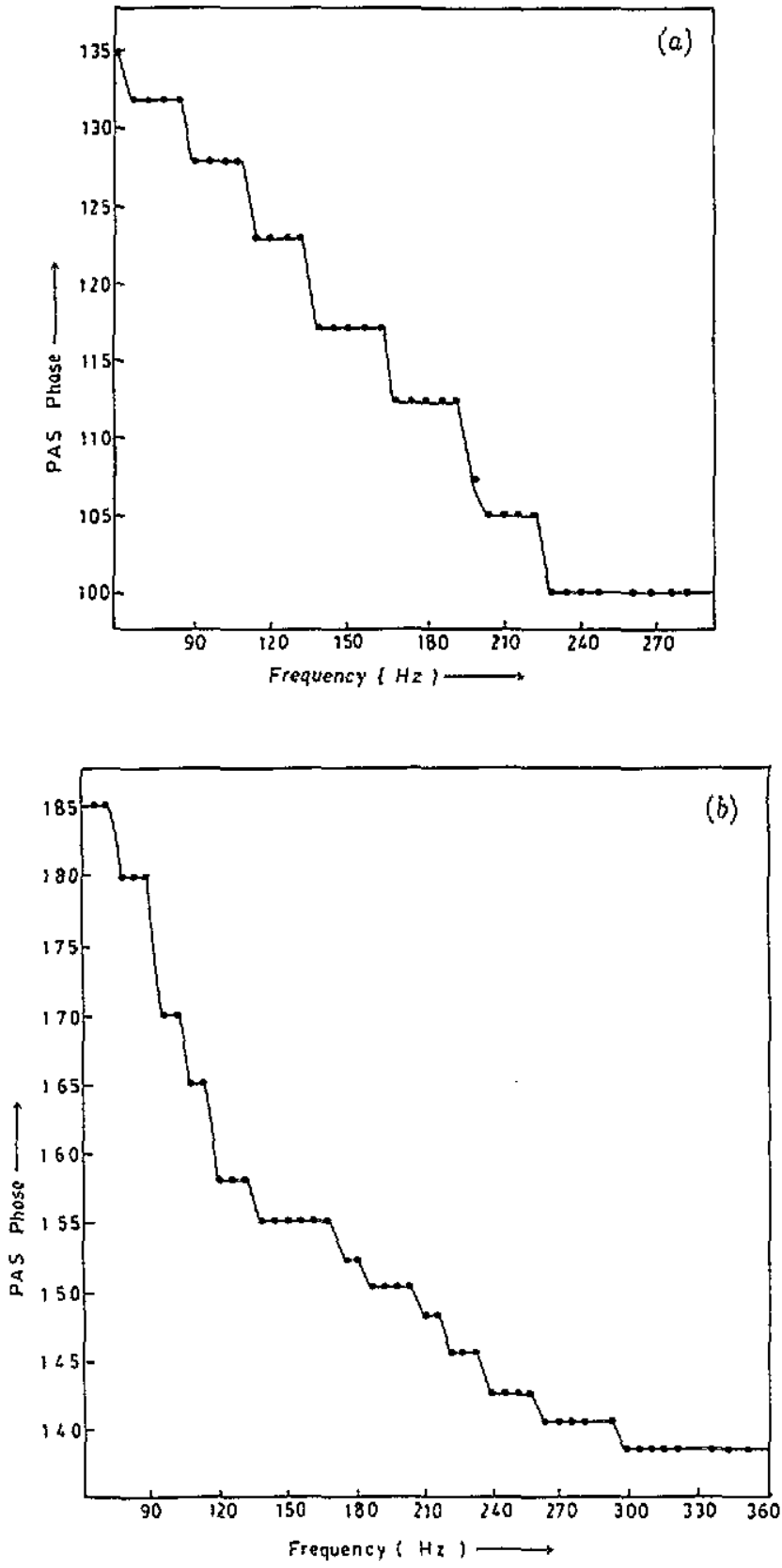

Figure 4. (a) Frequency against PA signal phase for 7 layer $\mathrm{SiO}_{2} / \mathrm{TiO}_{2}$ structure. (b) Frequency against PA signal phase for 13 layer $\mathrm{SiO}_{2} / \mathrm{TiO}_{2}$ structure.

\section{Acknowledgments}

We thank the National Physical Laboratory, New Delhi, for providing samples. Financial assistance from the Council of Scientific and Industrial Research and the Ministry of Human Resource Development is also gratefully acknowledged.

\section{References}

Adam M J and Kirkbright G F 1977 Analyst 102678 Baumann J and Tilgner R 1985 J. Appl. Phys. 581982 Mandelis A, Teng Y C and Royce B S H 1979 J. Appl. Phys. 507138

Opsal J and Rosencwaig A 1982 J. Appl. Phys. 534240 Tam A C. 1986 Rev. Mod. Phys. $\mathbf{5 8} 418$ 